\section{Targeting thrombolysis in pulmonary embolism}

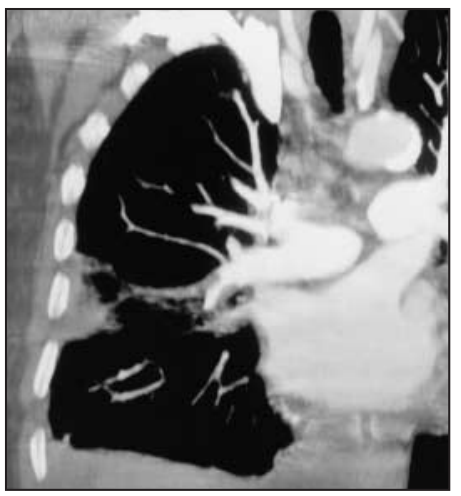

Systemic thrombolytic drugs are widely used to treat thrombotic diseases (e.g., stroke, myocardial infarction, pulmonary embolism). However, this treatment is associated with potentially serious bleeding complications because of the secondary consumption of systemic fibrinogen caused by the drug. Ding and colleagues present a new concept of tailoring thrombolytic drugs in the treatment of pulmonary embolism.

The authors, working with the premise that the anatomy of vascular tissue is distinct in various organs, identified an anti-

$\infty$ body that was specific to the $\hat{\sigma}$ vascular endothelium found in pulmonary tissue. They then coupled this antibody to the thrombolytic drug, urokinase.

Using a mouse model, they in$\ddot{\vec{B}}$ duced pulmonary embolism in vivo and demonstrated that clot lysis from 8000 units of the urokinase-antibody conjugate was similar to 100000 units of standard urokinase. They also demonstrated that, unlike the standard drug, the conjugate caused a minimal difference in bleeding times, which suggests that the tailored drug was delivered to the pulmonary vasculature with little breakdown of systemic fibrinogen. (Ding et al. Circulation 2003;108:2892)

\section{The autonomic system in sepsis}

Despite advances in antimicrobial therapies and critical care, sepsis remains the most frequent cause of death in intensive care units. Sepsis causes a systemic inflammatory response syndrome that results in cytokinemediated hypotension and multiple organ failure. There is evidence that cardiovascular autonomic failure plays an important role in the process.

Sharshar and colleagues performed postmortem analyses, comparing patients who had died of septic shock with those who had died of other causes of shock (cardiogenic, hemorrhagic). To determine whether autonomic failure was centrally mediated, they specifically looked at areas of the brain known to be involved in cardiovascular autonomic regulation, and areas known to be most sensitive to ischemia.

When they compared the ischemic-sensitive areas of the brain, they found similar ischemic changes in the septic shock and non-septic shock groups. However, there was a marked increase in apoptosis (cell death) in the autonomic centres in the septic shock group. The authors also measured inflammatory mediators and found increased levels of nitric oxide in the brains of the septic shock group.

These findings suggest that, although a similar degree of ischemia occurs in various types of shock, septic shock is characterized by increased apoptosis in areas of the brain crucial to cardiovascular autonomic control. Furthermore, the authors conclude that nitric oxide, rather than ischemia, may be the trigger for neuronal apoptosis. Their findings contribute to our understanding of the complex pathophysiology of sepsis that remains central to the development of future therapies. (Sharshar et al. Lancet 2003;362:1799)

- Compiled by Stephen Choi, 